\title{
Visualization of internalization of functionalized cobalt ferrite nanoparticles and their intracellular fate
}

This article was published in the following Dove Press journal:

International Journal of Nanomedicine

2 March 2013

Number of times this article has been viewed

\author{
Vladimir B Bregar ${ }^{1, *}$ \\ Jasna Lojk ${ }^{1, *}$ \\ Vid Šuštar ${ }^{1,2}$ \\ Peter Veranič ${ }^{2}$ \\ Mojca Pavlin' \\ 'Faculty of Electrical Engineering, \\ University of Ljubljana, Ljubljana, \\ Slovenia; ${ }^{2}$ Faculty of Medicine, \\ University of Ljubljana, Ljubljana, \\ Slovenia \\ *Both authors contributed equally to \\ this work
}

\begin{abstract}
In recent years, nanoparticles (NPs) and related applications have become an intensive area of research, especially in the biotechnological and biomedical fields, with magnetic NPs being one of the promising tools for tumor treatment and as MRI-contrast enhancers. Several internalization and cytotoxicity studies have been performed, but there are still many unanswered questions concerning NP interactions with cells and NP stability. In this study, we prepared functionalized magnetic NPs coated with polyacrylic acid, which were stable in physiological conditions and which were also nontoxic short-term. Using fluorescence, scanning, and transmission electron microscopy, we were able to observe and determine the internalization pathways of polyacrylic acid-coated NPs in Chinese hamster ovary cells. With scanning electron microscopy we captured what might be the first step of NPs internalization - an endocytic vesicle in the process of formation enclosing NPs bound to the membrane. With fluorescence microscopy we observed that NP aggregates were rapidly internalized, in a time-dependent manner, via macropinocytosis and clathrin-mediated endocytosis. Inside the cytoplasm, aggregated NPs were found enclosed in acidified vesicles accumulated in the perinuclear region 1 hour after exposure, where they stayed for up to 24 hours. High intracellular loading of NPs in the Chinese hamster ovary cells was obtained after 24 hours, with no observable toxic effects. Thus polyacrylic acid-coated NPs have potential for use in biotechnological and biomedical applications.
\end{abstract}

Keywords: internalization, magnetic nanoparticles, intracellular fate, transmission electron microscopy, scanning electron microscopy

\section{Introduction}

In recent years, nanoparticles (NPs) and related applications have become an intensive area of research due to their small size and advantageous properties compared with bulk materials. In our everyday life we are exposed to NPs through contact with products containing NPs and due to increasing environmental pollution. ${ }^{1,2}$ In the last decades, several applications utilizing NPs have also been developed in the fields of biotechnology and biomedicine. ${ }^{3,4}$

In biomedical fields, NPs are used for tissue engineering, tumor treatment, for contrast enhancement in magnetic resonance imaging (MRI), and for drug delivery. ${ }^{3,5}$ Several of these applications are already in clinical use. ${ }^{46}$ Different types of NPs are used, where in general, biodegradable NPs (eg, liposomes, polymers) are preferred for drug delivery and targeting tumors, thus eliminating possible side effects due to retention of nanocarriers. ${ }^{4,7}$ On the other hand, magnetic nanoparticles give us the possibility of their manipulation by a static or time-varying magnetic field. ${ }^{6}$ Currently, several types of magnetic NPs are used for magnetic separation, drug delivery, hyperthermic treatment of tumors, and as MRI-contrast agents. ${ }^{6,8}$
Correspondence: Mojca Pavlin Faculty of Electrical Engineering, University of Ljubljana, Trzaska 25, 1000 Ljubljana, Slovenia

Tel +386 I 4768949

Fax +386 I 4264850

Email mojca.pavlin@fe.uni-lj.si 
Despite considerable utilization of NPs, there are still many unanswered questions concerning their interactions with cells. Several cytotoxic and internalization studies have been done on different cell lines ${ }^{9,10}$ using different NPs in an attempt to determine the mechanisms of NP internalization and damage. It has been shown that several factors determine the internalization of NPs, including both cell physiology ${ }^{11}$ and NP properties (size, material, surface characteristics, and shape). ${ }^{12-15}$ Most NPs have been shown to enter the cells, in an energy-dependent way, ${ }^{16-20}$ through the process of endocytosis. ${ }^{21}$ In general, the intracellular fate of internalized particles is strongly affected by the route of entry. ${ }^{11}$

Internalization has also been intensively visualized, mostly by using fluorescently labeled NPs and their colocalization with several intracellular organelles. ${ }^{22}$ Once internalized, NPs are mostly reported to be present in vesicles ${ }^{11,16,18,23-25}$ that travel toward the perinuclear region. ${ }^{19,24,26-32}$ Most research groups report colocalization of fluorescently labeled NPs with lysosomes and late endosomes. ${ }^{9,20,29-31,33}$

Electron microscopy is also extensively used for NP research, mostly for NP characterization and analysis of surface characteristics. For this, both scanning (SEM) and transmission electron microscopy (TEM) are used, sometimes combined with X-ray spectroscopy. SEM is used for observation of morphological changes in cell membranes after incubation with NPs, ${ }^{34,35}$ but rarely for observations of NPs on the cell surface..$^{35}$ On the other hand, TEM is widely used for observation of internalized NPs inside cells, to determine the subcellular location of NPs and their aggregation. ${ }^{18,19,23,30,36}$ Despite the large number of internalization studies, only few have visualized the first steps of NP internalization interactions of NPs with the cell membrane.

The aim of our investigation was to visualize the first steps of the interaction of functionalized magnetic NPs (cobalt ferrite NPs coated with polyacrylic acid) with adherent Chinese hamster ovary (CHO) cells: the binding of NPs to the cell surface and the induction of endocytic internalization. We observed these processes using three different microscopic methods, fluorescence microscopy, SEM, and TEM. We also briefly looked into the intracellular fate of internalized NPs and their effect on cell viability and proliferation.

\section{Material and methods}

\section{Cell lines and cell culturing}

All experiments were done with $\mathrm{CHO}$ cells. Cells were grown in Ham's tissue culture medium for mammalian cells (HAM; Sigma-Aldrich, St Louis, MO, USA), supplemented with 10\% fetal bovine serum (FBS) (Sigma-Aldrich), $0.5 \%$ L-glutamine (Sigma-Aldrich), $0.1 \%$ gentamicin (PAA Laboratories, Pasching, Austria), and $0.01 \%$ penicillin (PAA Laboratories), at $37^{\circ} \mathrm{C}$ in a humidified $5 \% \mathrm{CO}_{2}$ atmosphere. All incubation experiments were performed on a 24-hour-old cell culture in HAM medium with $10 \%$ FBS.

\section{Nanoparticle synthesis and characterization}

Cobalt ferrite $\mathrm{CoFe}_{2} \mathrm{O}_{4}$ (Co-ferrite) NPs were prepared by coprecipitation ${ }^{37-39}$ and stabilized in water. After NPs were obtained, alkaline medium was removed and replaced with distilled water subsequent to magnetic decantation of the NPs (repeated three times), in order to obtain a stable dispersion of nanoparticles (ferrofluid). NPs were coated in situ with a $45 \%(\mathrm{w} / \mathrm{w})$ water solution of polyacrylic acid (PAA) sodium salt with molecular weight of $8 \mathrm{kDa}$ (Sigma-Aldrich), by mixing $10 \mathrm{~mL}$ of ferrofluid and $10 \mathrm{~mL}$ of the PAA water solution of equal mass concentrations at $20 \mathrm{mg} / \mathrm{mL}$, under magnetic stirring for 10 minutes at room temperature. ${ }^{37,40}$ Larger agglomerates were removed by centrifugation. For size characterization of the NPs, we used TEM (JEM 2100; JEOL Ltd, Tokyo, Japan) and dynamic light scattering (DLS) (Malvern Zetasizer Nano ZS; Malvern Industries, Malvern, UK) with the NIBS (noninvasive backscatter) $173^{\circ}$ backscatter algorithm. Zeta potential measurements were done with disposable folded capillary cells and the M3-PALS measurement technology, built in the Malvern Nanosizer NanoZS system.

\section{Cell viability and proliferation assay MTS viability assay}

CHO cells were grown in 96-well plates (Corning Inc, Corning, NY, USA) and incubated with different concentration of NPs $(50,100,150$, and $200 \mu \mathrm{g} / \mathrm{mL})$ for 24 hours. After incubation, NPs were removed by washing the cells three times with warmed HAM medium and were left in $100 \mu \mathrm{L}$ HAM per well. Then, $25 \mu \mathrm{L}$ of MTS reagent 3-(4,5-dimethylthiazol-2-yl)-5-(3-carboxymethoxyphenyl)-2-(4-sulfophenyl)2H-tetrazolium, inner salt (Cell Titer ${ }^{\circledR} 96$ AQueous One Solution Cell Proliferation Assay; Promega Corp, Fitchburg, WI, USA) was added per well and left to incubate another 3 hours. After incubation, $100 \mu \mathrm{L}$ of medium from each well was transferred into fresh wells to avoid the interference of internalized NPs with absorbance measurements. Absorbance was measured at $490 \mathrm{~nm}$ using the Tecan Infinite 200 (Tecan Group Ltd, Männedorf, Switzerland). The results are represented as the percentage of relative fluorescence units 
compared with the control, from all three experiments, with corresponding standard error.

\section{Propidium iodide viability assay}

Cells were grown in a 24 well-plate (Corning Inc) and incubated with different concentration of NPs $(50,100,150$, and $200 \mu \mathrm{g} / \mathrm{mL}$ ) for 24 hours. After incubation, cells were gently washed three times with warmed PBS and incubated with cell culture medium containing propidium iodide (PI) $(0.15 \mathrm{mM}$; Sigma-Aldrich) for 5 minutes, allowing the fluorescent dye to enter only dead cells (PI is membrane impermeable for short incubation times). After incubation, cells were washed three times with warmed PBS. For each sample, 10 visual fields (at $200 \times$ magnification) were taken, using phase contrast and fluorescence, and cells were counted (approximately 200 cells per visual field). The number of dead cells was subtracted from the total number of the cells. The experiment was repeated three times. The results are represented as percentage of cell number compared with the control, from all three experiments, with corresponding standard error.

\section{Fluorescence microscopy}

To observe the internalized NPs with fluorescent microscopy, red fluorescent dye rhodamine B isothiocyanate (RITC; Sigma-Aldrich) was electrostatically bound to the NPs. The NPs were mixed with RITC solution and dialyzed against distilled water for 24 hours. Cells incubated with a corresponding amount of last dialysate buffer were used as a control.

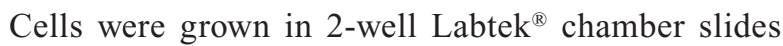
(Thermo Fisher Scientific Inc, Waltham, MA, USA) and incubated with $100 \mu \mathrm{g} / \mathrm{mL}$ NPs in HAM with $10 \%$ FBS for different time intervals $(15,30,45,60$, and 75 minutes, 24 hours). After the incubation, the medium with NPs was removed, and cells were washed three times with warmed PBS and observed using an inverted confocal microscope Leica TCS SP5 (Leica, Wetzlar, Germany). To colocalize the internalized NPs with lysosomes, cells were incubated with 75nM LysoTracker ${ }^{\circledR}$ Blue solution (Life Technologies Corp, Carlsbad, CA, USA) and dissolved in HAM with 10\% FBS, for at least 30 minutes.

Obtained fluorescent images were analyzed using ImageJ software (v1.45s, National Institute of Health, USA).

\section{TEM}

CHO cells were grown in 2-well Labtek chamber slides (Thermo Fisher Scientific Inc) to $40 \%$ confluency and incubated with $100 \mu \mathrm{g} / \mathrm{mL}$ NPs for different time intervals $(15,30$, 45, 60 minutes, and 24 hours). The appropriate solvent was added to the control samples. After incubation, the NPs were removed, and the cells were washed three times with warmed PBS and fixed with a mixture of $4 \%(\mathrm{w} / \mathrm{v})$ formaldehyde and $2 \%(\mathrm{w} / \mathrm{v})$ glutaraldehyde in $0.1 \mathrm{M}$ cacodylate buffer $(\mathrm{pH} 7.4)$, for 2 hours at room temperature. Postfixation was carried out in $1 \%$ osmium tetroxide in $0.1 \mathrm{M}$ cacodylate buffer for 2 hours, followed by dehydration in graded ethanol and embedding in Epon 812 resin (Electron Microscopy Sciences, Hatfield, PA, USA). Ultrathin sections were counterstained with uranyl acetate and lead citrate and examined with TEM (CM100; Royal Philips Electronics, Amsterdam, The Netherlands).

\section{SEM}

CHO cells were grown in 2-well Labtek chamber slides (Thermo Fisher Scientific Inc) to $40 \%$ confluency and incubated with $100 \mu \mathrm{g} / \mathrm{mL}$ NPs for different time intervals $(5,10,20,30$, and 60 minutes $)$ at $37^{\circ} \mathrm{C}$. The appropriate solvent was added to the control. After incubation, the NPs were removed, and the cells were washed three times with warmed PBS and fixed with a mixture of $4 \%(w / v)$ formaldehyde and $2 \%(\mathrm{w} / \mathrm{v})$ glutaraldehyde in $0.1 \mathrm{M}$ cacodylate buffer for 2 hours. Samples were washed with cacodylate buffer and postfixated in 4\% osmium tetroxide for 1 hour; washed again and incubated with 1\% tannic acid (Electron Microscopy Sciences) in $0.05 \mathrm{M}$ cacodylate buffer for 30 minutes; followed by washing in cacodylate buffer and dehydration in progressive series of ethanol, which was exchanged in a progressive series with hexamethyldisilazane (HMDS) (Structure Probe Inc, West Chester, PA, USA); and incubated overnight. The samples in HMDS were air dried and carbon sputtered. SEM and X-ray analysis were performed with LEO Gemini 1530 scanning electron microscope (LEO Electron Microscopy Ltd, Oberkochen, Germany) with inbuilt X-ray UltraDry Silicon Drift Detector (Thermo Fisher Scientific). The whole fixation was carried out at room temperature.

\section{Results}

We synthesized stable magnetic NPs coated with PAA. Here we present results showing the first steps of internalization of our NPs, obtained by using TEM, SEM, and fluorescence microscopy. We furthermore observed the intracellular fate of internalized NPs and their effect on cell viability.

\section{Nanoparticle characterization}

The size of synthesized Co-ferrite NPs was characterized using TEM, which enabled us to measure the crystalline size, while DLS measurements of the hydrodynamic diameter gave us the information of the effective NP size in the 


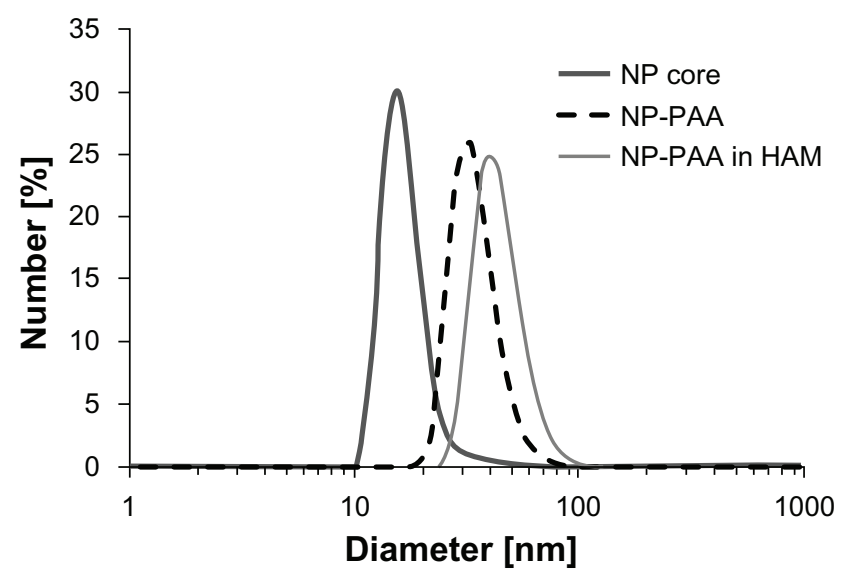

Figure I Dynamic light scattering analysis of Co-ferrite NPs.

Notes: Distribution of the hydrodynamic diameters is shown for: NP cores in distilled water, NPs functionalized with PAA in distilled water, and functionalized NPs in cell culture medium with $10 \%$ FBS, which was used for all internalization experiments.

Abbreviations: Co-ferrite, cobalt ferrite; NP, nanoparticle; PAA, polyacrylic acid; HAM, Ham's tissue culture medium for mammalian cells; FBS, fetal bovine serum.

suspension. Using DLS, the measured average diameter of the nonfunctionalized NP core was $16 \mathrm{~nm}$ (Figure 1), while TEM gave the average diameter of $10 \mathrm{~nm}$ (Figure 2). This implies that NPs in suspension are composed of a few primary crystallites forming the agglomerate. Since these agglomerates cannot be divided and behave as a single particle, individual agglomerates will be referred to as nanoparticles (nanoparticle cores). These NPs were functionalized with PAA, resulting in particles with long-term high stability in

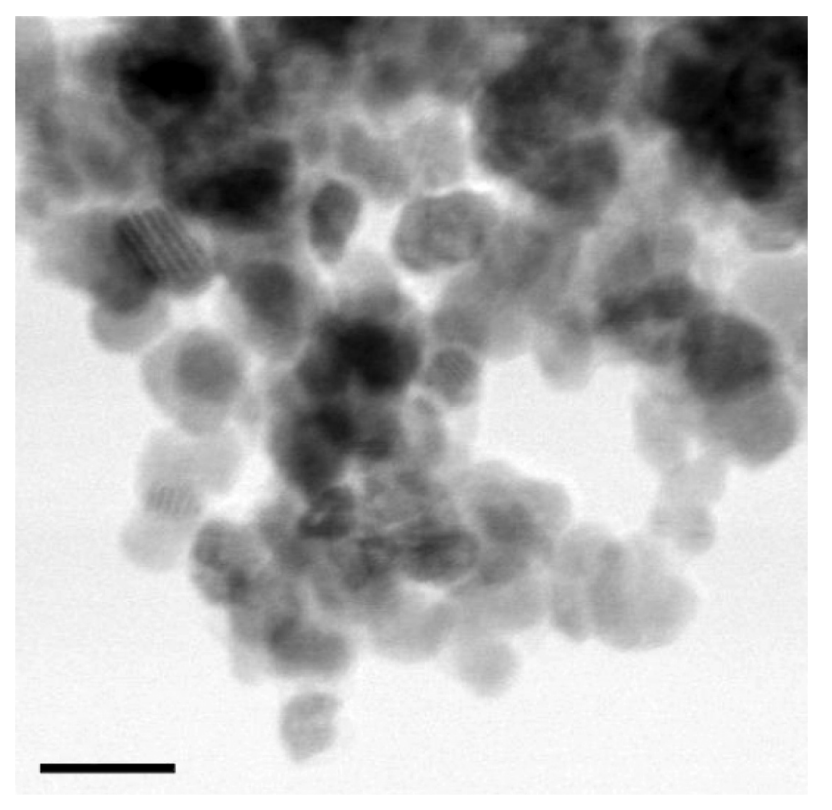

Figure 2 TEM micrograph of Co-ferrite NP cores.

Notes: The average diameter of NPs is $10 \mathrm{~nm}$. The Scale bar corresponds to $20 \mathrm{~nm}$. Abbreviations: TEM, transmission electron microscopy; Co-ferrite, cobalt ferrite; NP, nanoparticle. water solution, and with an average diameter of $33 \mathrm{~nm}$. Since all experiments were performed in cell culture medium, we also characterized NPs suspended in HAM supplemented with $10 \%$ FBS. The average diameter of the NPs in HAM with $10 \%$ FBS was $42 \mathrm{~nm}$, probably due mainly to the binding of serum proteins and partially due to aggregation. ${ }^{41}$ PAA-coated NPs in HAM cell culture medium were relatively stable: the nanoparticle suspension was not stable over longer periods of time (days), but agglomeration was not very strong, as shown in Figure 1, and a significant part of NPs suspension was still dispersed. The zeta potential of PAA-coated NPs in water suspension at $\mathrm{pH}=7.7$ was $\zeta=-50 \mathrm{mV}$ and in HAM with $10 \%$ FBS was approximately $\zeta=-17 \mathrm{mV}$.

\section{Nanoparticle-membrane interactions and internalization}

CHO cells were incubated with Co-ferrite NPs for different time intervals and the first steps of internalization were observed using SEM and TEM. After 1 hour of incubation, control cells showed normal shape and surface morphology under SEM (Figure 3A). These cells were normally spread, with lamellipodia and several randomly distributed filopodia present on the cell surface (Figure 3C). Conversely, exposure to NPs induced perturbations in the cell surface membrane (Figure 3B) similar to those observed during macropinocytosis. ${ }^{42}$ Three types of membrane ruffling were already observed after 10 minute incubation, mostly blebs (Figure 3D), but also circular ruffles (Figure 3E) and planar lamellipodia (Figure 3F). Similar membrane disruptions were also observed after longer incubation times (20 minutes, 30 minutes, and 60 minutes) (data not shown), indicating that cells immediately respond to the presence of NPs and that the internalization continues for at least up to 1 hour from the moment of exposure. NP aggregates were observed bound to these membrane disruptions or present in their vicinity (Figures 3F and 4), as confirmed with X-ray spectroscopy (Figure 5). Also, a slightly increased number of nanotubes were observed between cells exposed to NPs (Figure 3B, denoted by arrows), as has already been shown for other NP types. ${ }^{43}$

In order to observe the interaction of the NPs with the cell membrane and to capture the moment of NP internalization, SEM was performed. We were able to visualize NPs present in a cavity with a diameter of approximately $500 \mathrm{~nm}$ in the outer cell membrane, which could represent the beginning of NP internalization (Figure 4). This was confirmed with X-ray elemental analysis (Figure 5), which detected the presence of iron in the NPs aggregates, shown in Figure 4. 

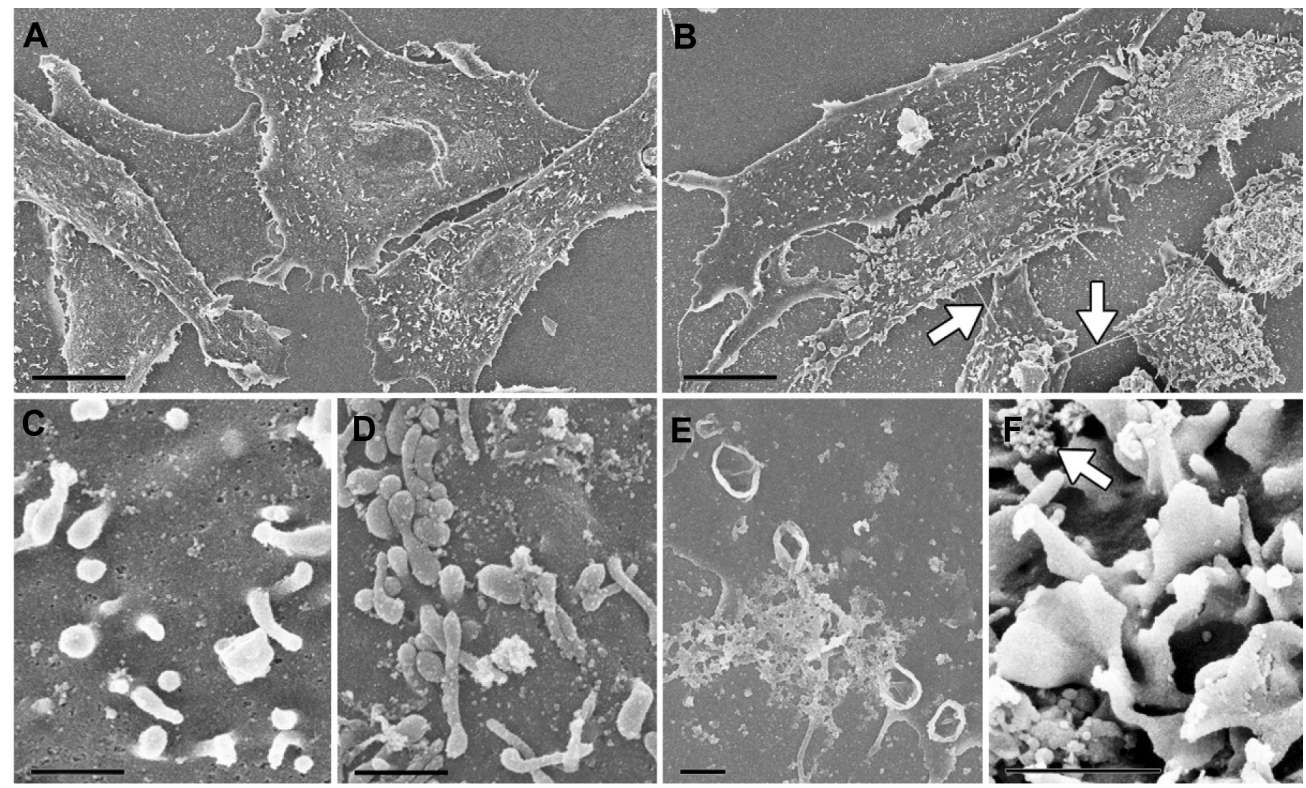

Figure 3 Exposure to functionalized Co-ferrite NPs induces membrane ruffling in $\mathrm{CHO}$ cells. CHO cells were incubated for I hour with distilled water (control) (A), or treated with NPs (B). (C-F), CHO cells were for 10 min incubated with distilled water (C), or with NPs and fixed. The presence of NPs induced perturbations of the plasma membrane, mostly in form of spherical membrane extensions (blebs) (D), circular ruffling, which look like cup-shaped projections (E), and lamellipodial ruffles, which appear as flat sheet-like projections on the celi surface $(\mathbf{F})$.

Notes: Arrows in panel (B) denote nantubes and in panel (F) NP aggregates. The scale bars correspond to $10 \mu \mathrm{m}$ in $(\mathbf{A})$ and (B), and to I $\mu \mathrm{m}$ in $(\mathbf{C}-\mathbf{F})$.

Abbreviations: Co-ferrite, cobalt ferrite; NP, nanoparticle; $\mathrm{CHO}$, Chinese hamster ovary.

In Figures 6 and 7, we present the process of NP internalization observed with TEM. Figure 6A shows membrane ruffles encircling NP aggregates bound to the outer cell membrane and in an already internalized vesicle. Membrane ruffles resemble the membrane perturbations typical for macropinocytosis. ${ }^{42}$ Also, already internalized NP

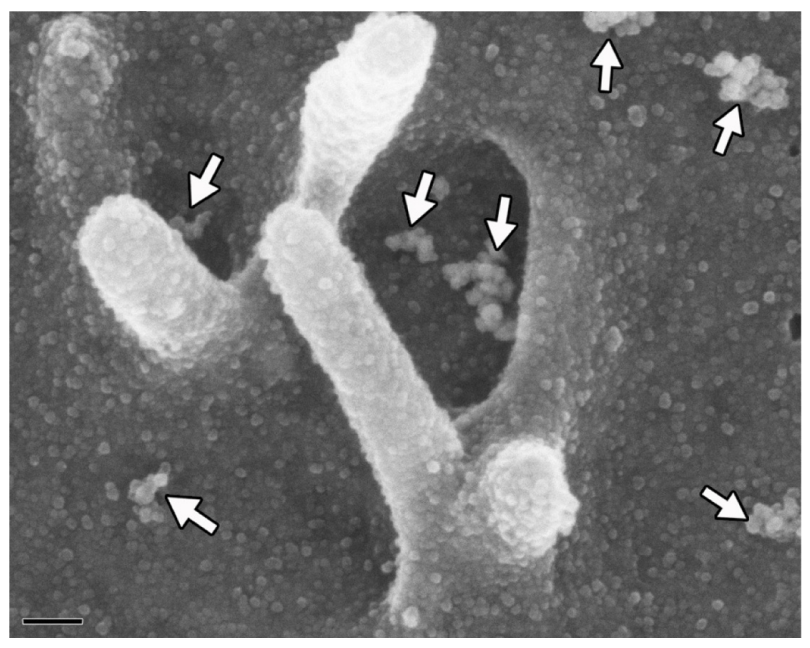

Figure 4 Interaction of functionalized Co-ferrite NPs with the cell membrane surface, observed with SEM.

Notes: $\mathrm{CHO}$ cells were incubated with NPs for 30 minutes and fixed. NP aggregates, bound to the plasma membrane are denoted by arrows, and some NPs are found in a cavity in the outer cell membrane. Individual nanoparticle crystallites can be observed. The scale bar corresponds to $100 \mathrm{~nm}$.

Abbreviations: Co-ferrite, cobalt ferrite; NP, nanoparticle; SEM, scanning electron microscopy; $\mathrm{CHO}$, Chinese hamster ovary. aggregates in early endosomes and in multivesicular bodies (late endosomes) were observed (Figure 6B).

In addition to macropinocytosis, NP aggregates were also observed in clathrin-coated pits (CCP) (Figure 7A) on the membrane surface and in already internalized clathrincoated vesicles (CCV) (Figure 7B, Figure S1). Membrane ruffles typical for macropinocytosis and $\mathrm{CCP}$ and $\mathrm{CCV}$ were observed for different times of incubation with NPs, and the most representative figures are shown.

Altogether, our SEM and TEM results indicate that functionalized Co-ferrite NP aggregates are internalized in $\mathrm{CHO}$ cells via at least two distinct internalization mechanisms simultaneously, macropinocytosis and clathrin-mediated endocytosis (CME). The occurrence of macropinocytotic uptake was much higher, and also, larger amounts of NPs were observed to enter the cells via macropinocytosis compared to $\mathrm{CME}$.

\section{Intracellular fate of nanoparticles}

We further analyzed the intracellular fate of NPs using TEM and fluorescence imaging of live cells. Consistent with the observed mechanisms of internalization, NPs were found in membrane-bound vesicles and in lysosomes, as shown in Figure 8. No NPs were found free in the cytoplasm or associated with other cell organelles, like the nucleus, endoplasmic reticulum, Golgi apparatus, or mitochondria, for all analyzed incubation times. 

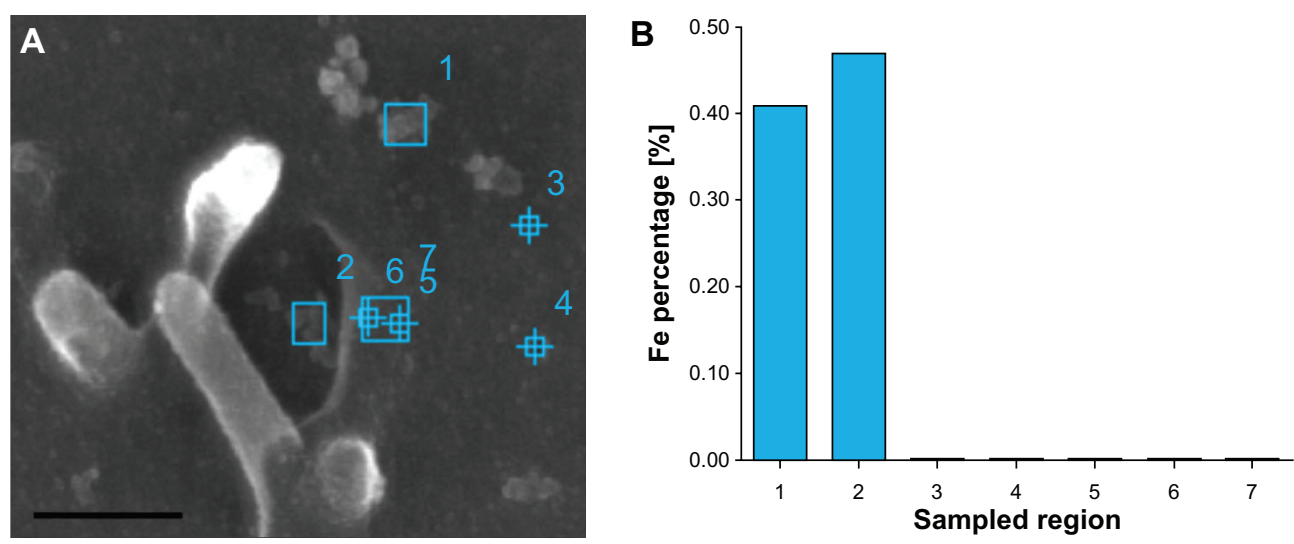

Figure 5 Energy-dispersive X-ray spectroscopy confirmed the presence of NP aggregates on the membrane of $\mathrm{CHO}$ cells. CHO cells were incubated with NPs for 30 minutes and fixed. In panel (A) there are marked and numbered areas, which correspond to sample regions in panel (B), values are represented as percentage of iron in the total elemental composition of the analysed region.

Note: The scale bar corresponds to $500 \mathrm{~nm}$.

Abbreviations: NP, nanoparticle; $\mathrm{CHO}$, Chinese hamster ovary.

In Figure 9, we present fluorescence images of live cells incubated with RITC-labeled Co-ferrite NPs and their colocalization with lysosomes and late endosomes labeled with LysoTracker ${ }^{\circledR}$ Blue, after 15 minutes (Figure 9A), 75 minutes (Figure 9B), and 24 hours (Figure 9C) of incubation with NPs, obtained with confocal microscopy. In agreement with SEM and TEM, NPs are already present on the cell surface and in primary vesicles, in the form of small aggregates, after 15 minutes of incubation (Figure 9A). Fluorescence images after 15 minutes of incubation show that first vesicles containing NPs already acidified, as indicated by colocalization with LysoTracker ${ }^{\circledR}$ Blue staining, but most NPs were still bound to the surface (Figure 9A). With longer incubation time, the number of internalized NP aggregates increased, and vesicles containing the NPs increased in size and accumulated in the perinuclear region of the cells (Figure 9B). Similar observations have also
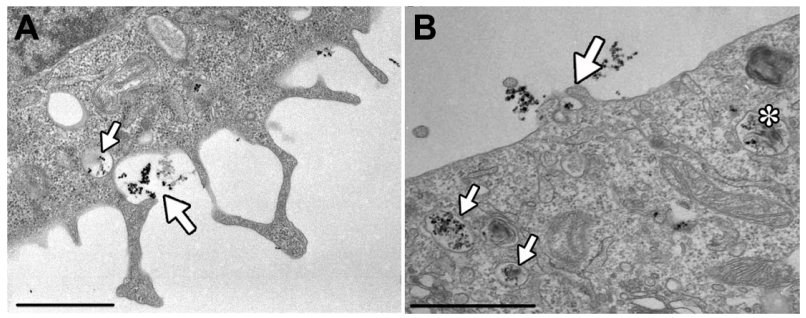

Figure 6 Internalization of Co-ferrite NPs in CHO cells observed with TEM. $\mathrm{CHO}$ cells were incubated with NPs for 50 minutes and fixed. Membrane ruffles observed with TEM resemble membrane perturbations typical of macropinocytosis (A and B).

Notes: NP aggregates are observed under membrane ruffles (big arrow) and in an already internalized vesicle (small arrow), most probably formed with macropinocytosis (A); membrane ruffles enclosing a small NP aggregate (big arrow) and already internalized NP aggregates in early endosomes (small arrows) and in a multivesicular body (asterisk) (B). The scale bars correspond to I $\mu \mathrm{m}$.

Abbreviations: Co-ferrite, cobalt ferrite; NP, nanoparticle; $\mathrm{CHO}$, Chinese hamster ovary; TEM, transmission electron microscopy. been reported for PAA particles in macrophages. ${ }^{44}$ After 75 minutes of incubation, most NPs were present in acidified late endosomes and lysosomes, as seen in Figure 9B; however, after 24 hours of incubation (Figure 9C), vesicles containing NPs were mostly no longer acidic, as observed by the lack of colocalization with LysoTracker ${ }^{\circledR}$ Blue staining. The fluorescence intensity of RITC-labeled NPs in the cytoplasm and the maximal fluorescence intensity of spots representing internalized NPs increased with longer incubation times (Figure 9 and Figure S2). These observations were also confirmed by a TEM micrograph of cells incubated with NPs for 24 hours (Figure 10), which show large NP aggregates inside the cytoplasm, mostly in the perinuclear region. Most of the aggregates, independently of their size, are present in vesicles (Figure 10, small arrows), but since in certain parts of the image the vesicle membrane is hardly or not visible, certain
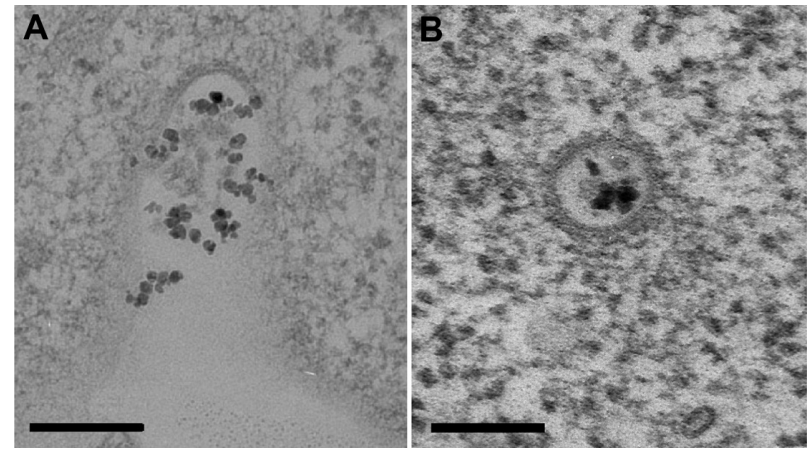

Figure 7 Functionalized Co-ferrite NPs enter $\mathrm{CHO}$ cells via clathrin-mediated endocytosis. Cells were incubated with NPs for 15 minutes and fixed. Small NP aggregates are present in a clathrin-coated pits on the cell surface $(\mathbf{A})$, and in a clathrin-coated vesicle immediately after internalization (B).

Notes: Presented figures are the most representative images of several clathrincoated pits and vesicles observed. The scale bars correspond to $200 \mathrm{~nm}$.

Abbreviations: Co-ferrite, cobalt ferrite; NP, nanoparticle; $\mathrm{CHO}$, Chinese hamster ovary. 


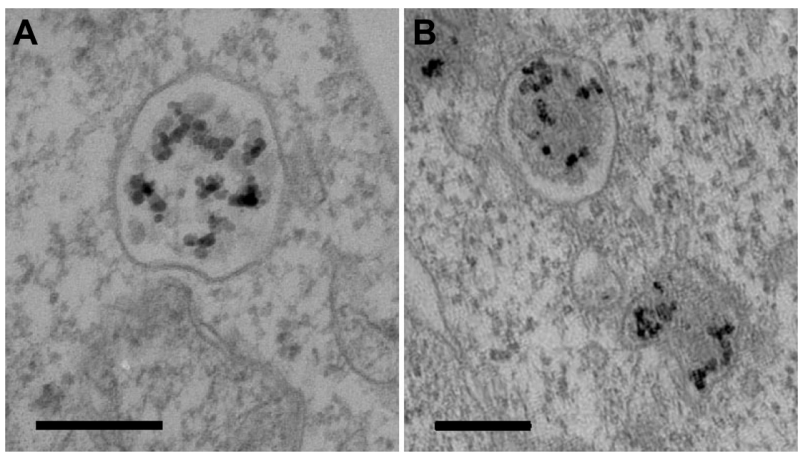

Figure 8 TEM observation of Co-ferrite NPs after internalization. Cells were incubated for I hour and fixed. NPs are found in larger aggregates in bigger membrane bound vesicles (A), and in lysosomes (B).

Note: The scale bars correspond to $200 \mathrm{~nm}$.

Abbreviations: TEM, transmission electron microscopy; Co-ferrite, cobalt ferrite; NP, nanoparticle.

NP aggregates could, as well, have escaped the vesicles and be present in the cytosol. No NPs were found in the nucleus nor associated with other intracellular organelles. Despite large quantities of internalized NPs, internalization was still observed (Figure 10A, big arrow).

\section{Effect of NP concentration on cell viability and proliferation}

To determine if observed intracellular retention of NPs after 24 hours of NP exposure (Figures 9C and 10) affects cell viability and proliferation, a viability and proliferation assay (Figure 11) was performed to determine the short-term cytotoxicity of NPs.

$\mathrm{CHO}$ cells were grown in the presence of four different concentrations of NPs for 24 hours, during which time the cells internalized NPs and divided. Two separate sets of experiments were performed: the first using an MTS viability assay; and the second using microscopy in combination with PI, to determine the percentage of viable cells. The standard MTS viability assay was performed according to the manufacturer's instructions. For PI viability assay, after 24 hours of incubation NPs were removed and cells were incubated for 5 minutes with PI, allowing the dye to enter and stain the cytoplasm of dead cells or cells with damaged plasma membranes (Figure S3). Cells were counted using phase contrast and fluorescence, which enabled us to determine the percentage of dead and viable cells. Cells in the control sample were not exposed to NPs. The 24-hour incubation with NPs had no effect on cell viability or on the rate of cell proliferation (Figure 11). PAA-functionalized magnetic NPs therefore induced no significant immediate toxicity and had no significant effect on cell viability or proliferation.

\section{Discussion}

Despite several internalization and cytotoxicity studies of nanoparticles (NPs) only a few studies have directly visualized the interaction of NPs with the cell membrane.

In our research we have focused on visualization of NP interactions with the cell membrane, their internalization, and intracellular fate in adherent $\mathrm{CHO}$ cells, using PAA-functionalized magnetic NPs. We synthesized PAAcoated Co-ferrite NPs, which have excellent dispersibility and long-term stability. PAA is a biocompatible anionic polymer, which enables very good stabilization of NPs in water-based suspensions, even at the relatively high ionic strengths encountered in cell culture media. ${ }^{41}$ PAA polymer
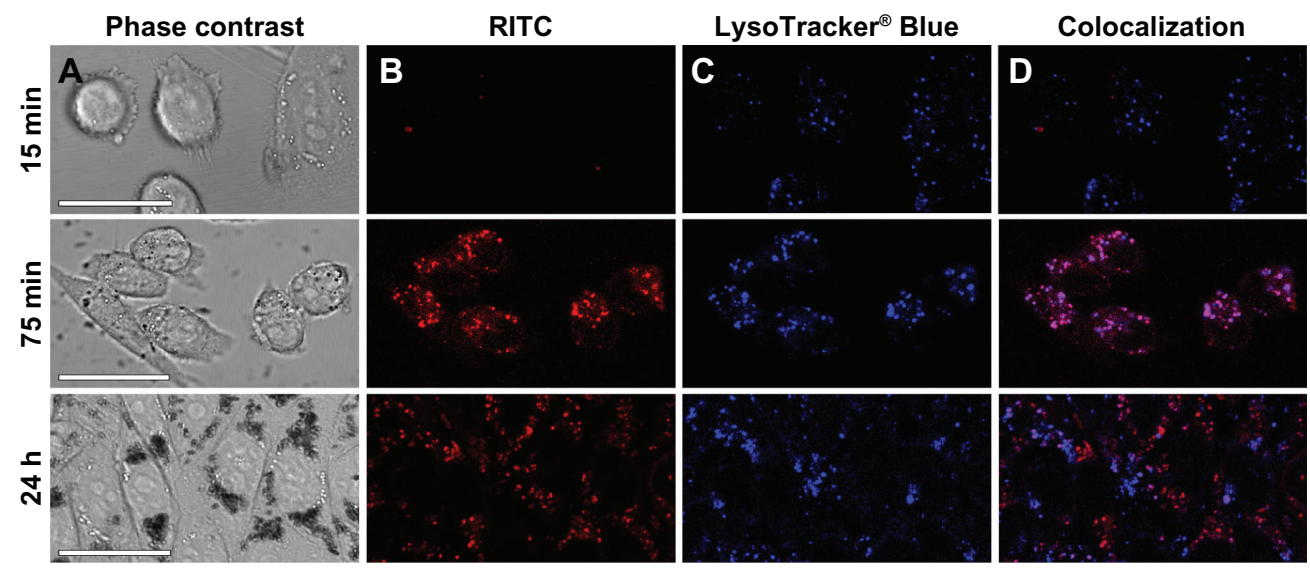

Figure 9 Time dependent internalization of Co-ferrite NPs: cells under phase contrast microscope (A), RITC labelled NPs (B), late endosomes and lysosomes labelled with LysoTracker ${ }^{\circledR}$ Blue (C) and colocalization of RITC labelled NPs and lysosomes (D) after 15 minutes, 75 minutes and 24 hours of incubation with NPs.

Notes: NPs can be observed inside the cells already after 15 minutes, but they are mostly not yet colocalized with lysosomes. After 75 minutes of incubation, colocalization of NPs and lysosomes is observed. After 24 hours of incubation, fewer vesicles containing NPs are colocalized with LysoTracker ${ }^{\circledR}$ Blue staining, suggesting that these vesicles are mostly no longer acidic. Presented figures are images of live cells. The scale bars correspond to $25 \mu \mathrm{m}$.

Abbreviations: RITC, rhodamine B isothiocyanate; Co-ferrite, cobalt ferrite; NP, nanoparticle. 


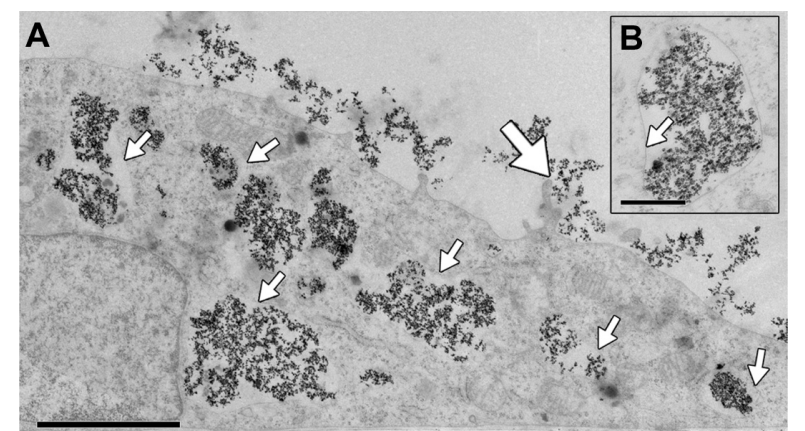

Figure 10 TEM micrograph of $\mathrm{CHO}$ cells incubated with Co-ferrite NPs for 24 hours. Big NP aggregates are mostly bound with membrane (small arrows) $(\mathbf{A}$ and $\mathbf{B})$ and accumulated in the perinuclear region $(\mathbf{A})$. Some membrane ruffling can be observed (big arrow), suggesting internalization (A).

Note: The scale bar corresponds to $2 \mu \mathrm{m}$ in (A) and to $0.5 \mu \mathrm{m}$ in (B).

Abbreviations: TEM, transmission electron microscopy; $\mathrm{CHO}$, Chinese hamster ovary; Co-ferrite, cobalt ferrite; NP, nanoparticle.

was chosen because it exhibits good biocompatibility together with excellent surface charge stabilization and good NP interactions and allows further functionalization. Accordingly, we observed that our PAA-functionalized magnetic NPs were nontoxic following short-term exposure (Figure 10), which is in agreement with several other studies. ${ }^{4-47}$ PAA-coated NPs were still very stable in the presence of HAM cell culture medium containing $10 \%$ FBS, resulting in NPs with an average diameter of $42 \mathrm{~nm}$, which was slightly larger than the same NPs in water $(33 \mathrm{~nm})$, as shown in Figure 1. This is due to protein adsorption and partially due to aggregation in the medium. ${ }^{41}$ Proteins and

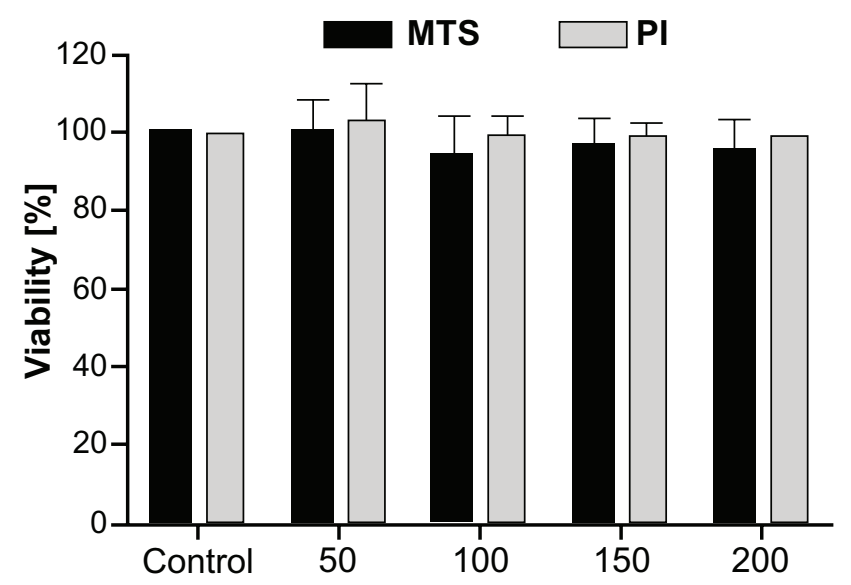

Nanoparticle concentration $[\mu \mathrm{g} / \mathrm{mL}]$

Figure II Viability and proliferation of cells after a 24-hour exposure to NPs, determined with MTS and PI viability assay.

Notes: Cells were incubated with increasing concentrations of functionalized NPs for 24 hours. The standard MTS viability assay was performed; for the PI viability assay, cells were incubated for 5 minutes with PI to detect dead cells. The results are presented as percentage of viable cells compared with the number of cells in the control sample $(\mathrm{Pl})$ or percentage viability determined with spectrofluorimetry (MTS). Mean and standard error are shown for three independent experiments. Abbreviations: NP, nanoparticle; MTS, MTS viability assay; PI, propidium iodide. divalent cations electrostatically bind to the negative charged surface of the NPs, effectively screening the stabilizing negative charge of the NPs and causing some aggregation. Adsorption of serum protein also explains the change of zeta potential from $\zeta=-50 \mathrm{mV}$ in the water suspension to $\zeta=-17 \mathrm{mV}$ in HAM with FBS. When NPs were incubated with cells, small aggregates formed, as seen on SEM (Figure 4) and TEM (Figure 6) micrographs, but most of them were still small enough to be internalized by cells via nonphagocytic pathways. ${ }^{21}$ Although PAA-coated NPs in cell culture medium were not stable over longer periods of time (days), agglomeration was not strong (Figures 6-8) and a dispersed fraction was always present.

The incubation of NPs with CHO cells in refreshed HAM with $10 \%$ FBS caused morphological changes on the cell surface, as shown in Figure 3. Cells became less spread (Figure 3B) but still attached, and since there was no significant effect of NPs on the rate of cell proliferation and viability after 24 hours (Figure 10), we assume that the membrane blebbing was caused by temporal stress due to medium change and the presence of NPs. In control cells, which also responded with the formation of blebs after medium change prior to incubation with the corresponding amount of appropriate solvent, cell morphology slowly returned to normal (as prior to medium change) (Figure 3A), while in cells incubated with NPs, the membrane perturbations were present for the entire time of incubation (Figure 3B). The persistent membrane blebbing is most probably due to the intensive internalization activity of the cells. The observed perturbations in the outer cell membrane resemble those described for macropinocytosis, ${ }^{42}$ blebs (Figure 3D), circular ruffles (Figure 3E), and planar lamellipodia (Figure 3F). Also, cells responded to NP presence with different intensity (Figure 3B), with shapes from almost spherical to normally spread, most probably due to different endocytosis rates in different cell cycle stages. ${ }^{48,49}$

Furthermore, using SEM also enabled us to visualize the interaction of NPs with the cell membrane. We were able to observe NPs present in a membrane cavity (Figure 4), which might represent the beginning of internalization of NP aggregates. Using X-ray elemental analysis, we confirmed the presence of Co-ferrite NPs, by detecting iron in the structures resembling NPs, inside the cavity (Figure 5).

Furthermore, combining SEM and TEM microscopy, we analyzed possible endocytic routes of NP entry into the cells. In general, NP aggregates were observed in the vicinity or under the membrane protrusions (Figure 6), making macropinocytosis a highly plausible pathway of NP 
internalization. Macropinocytosis is a non-receptor-mediated endocytic mechanism that enables the internalization of larger volumes and also particles, into nonphagocytic cells. Since the formation of macropinosomes (endocytic vesicles formed with macropinocytosis) is not guided by coat proteins, macropinosomes can be heterogeneous in size and shape, also reaching $10 \mu \mathrm{m}$ in diameter. ${ }^{42,50}$ This mechanism thus also enables the nonspecific internalization of large NP aggregates, as seen in Figure 6A.

The second observed internalization pathway was CME. CME is a receptor-mediated endocytic pathway, responsible for the internalization of several activated signaling receptors and also for the uptake of nutrients. Characteristic of this endocytic pathway is a clathrin coat, which drives the formation of CCP on the plasma membrane and stabilizes the CCV. Due to the geometry of the coat, these vesicles can only be 100-200 $\mathrm{nm}$ in diameter, ${ }^{21}$ thus enabling only internalization of smaller NP aggregates. We observed NP aggregates in CCP on the plasma membrane (Figure 7A) and also NPs enclosed in a CCV (Figure 7B), thus confirming the internalization of NPs with this endocytic pathway. Since the CCV are small, only a small amount of unspecific cargo in the lumen of the vesicle is taken in, suggesting that the NPs internalized via CME are recruited and retained in the CCP by binding to the appropriate receptors in the pits (via serum proteins that are electrostatically bound to the surface of the NPs) ${ }^{51}$ Here we have to stress that since only smaller aggregates of NPs can enter the cells via CME, most of NPs enter the cells via macropinocytosis, but further experiments would be needed in order to quantify the contribution of each endocytic pathway to internalization.

Several NP internalization studies have been done on $\mathrm{CHO}$ cells so far, all demonstrating active, energy-dependent type of internalization and locating NPs to intracellular vesicles, ${ }^{11,52,53}$ which is consistent with our results. Positively charged alginate-chitosan NPs have been shown to enter the cells via caveolin-mediated endocytosis, ${ }^{11}$ and large aggregates of $\mathrm{TiO}_{2}$ and $\mathrm{Al}_{2} \mathrm{O}_{3}$ were proposed to enter via phagocytosis or possibly via macropinocytosis, resulting in altered cell morphology. ${ }^{52} \mathrm{CME}$ was also previously observed, in $\mathrm{CHO}$ cells, as a pathway for the internalization of $100 \mathrm{~nm}$ NPs, targeted to transferrin receptors. So far, only one endocytic pathway has been reported for the internalization of certain type of NPs, but NP internalization via more than one pathway at the same time has been reported for other cell lines. ${ }^{30}$
Using TEM and SEM, we could observe the internalization of NPs in CHO cells, without the use of different inhibitors for specific endocytic pathways; these inhibitors are convenient to analyze different routes of endocytosis, however, they are not $100 \%$ effective and can possibly affect other cell processes, thus uncontrollably affecting normal cell functions. ${ }^{54-56}$ Using microscopic techniques enabled us to observe normally functioning cells and to reliably qualitatively determine endocytosis pathways, although not also quantitatively.

Here we have to stress that our study was limited to a single cell line and to one type of magnetic NP coated with PAA. Since it has been shown in several studies that the interaction of NPs with cells and their entry depends both on NP characteristics and cell line, ${ }^{11-15}$ further studies are necessary in order to determine if these are general entry pathways for PAA-coated NPs.

Furthermore, using fluorescence microscopy, we could observe that the internalization of our PAA-coated magnetic NPs was a fast process, since some NPs were already observed in the cytoplasm after 15 minutes of incubation (Figure 9A). This was also confirmed by TEM (results not shown). After internalization, NPs enclosed in membrane-bound vesicles travel towards and accumulate in the perinuclear region, as observed at longer NPs incubation times (Figure 9B). Endosomes containing NPs fuse with lysosomes, ${ }^{57}$ as seen by colocalization with LysoTracker ${ }^{\circledR}$ Blue (Figure 9B) after 75 minutes of NP incubation. Our results are in agreement with the results of several others. ${ }^{19,20,24,26-31,33}$ Despite the fact that the cells have mechanisms to secrete the internalized cargo as observed previously, ${ }^{17,26,36,58} \mathrm{CHO}$ cells seem to combine early endosomes into larger late endosomes and accumulate them in the perinuclear region (Figure 10), which has also been observed for other cell types. ${ }^{11,23,27,32}$ Aggregations in the perinuclear region are probably a result of endosomal fusions, combining several vesicles with small aggregates, as suggested by the observed bigger aggregates after 24 hours (Figure 10) and the spontaneous aggregations of NPs in acid environment. ${ }^{33}$ Since PAA has its isoelectric point at about $\mathrm{pH} 2$, its negative surface charge is reduced in acidic lysosomes, which consequently decreases NP stability, leading to aggregation. ${ }^{41}$

In addition to the increased number of fluorescent spots and their increasing size with longer incubation times, we also observed an increase in the background fluorescence of the cytoplasm and an increase in the maximal fluorescence intensity of spots representing internalized NPs (Figures 9A and B, Figure S2). The increase in cytoplasm 
fluorescence intensity can be partially attributed to the release of electrostatically bound RITC dye from NPs and partially due to the larger number of internalized NPs. The increase in maximum intensity is most probably due to the formation of larger aggregates of NPs from smaller internalized NP aggregates, in turn due to endosome fusion and the acidification of lysosomes. ${ }^{33}$

In order to further analyze the intracellular fate of our NPs, we incubated CHO cells with NPs for 24 hours. We could observe large NP aggregates, possibly inside vesicles, as large, intensively fluorescent spots (Figure 9C), which also indicates that lysosome enzymes did not degrade the PAA coating (which would otherwise result in RITC detachment and staining of the cytoplasm). Moreover, after 24 hours, these vesicles were mostly no longer acidic, avoiding further degradation, as seen from Figure 9C where after 24 hours, almost no colocalization between fluorescent NPs and LysoTracker ${ }^{\circledR}$ Blue was obtained. Furthermore, nonfunctionalized Co-ferrite NPs have been shown to be nontoxic after 24 hours at similar concentrations. ${ }^{32}$

To conclude, we have developed PAA-coated magnetic NPs. Polymeric coating enables good stability in physiological conditions, biocompatibility, and further functionalization. Using three types of microscopic techniques - TEM, SEM and fluorescence microscopy - enabled us to visualize the process of NP internalization. Results obtained by SEM and TEM suggest that our PAA-coated magnetic NPs are taken up by two endocytic pathways: clathrin-mediated endocytosis and macropinocytosis. Using scanning electron microscopy we could observe NP interactions with the plasma cell membrane at the initial stage of possible NP internalization. Furthermore, we observed the intracellular fate of NP aggregates, which were rapidly internalized and were still present in vesicles localized in the perinuclear region after 24 hours. Our results show that PAA-coated NPs are potentially interesting for use in biotechnological and biomedical applications, since no cytotoxic effects were observed after a 24-hour incubation. However, further studies are needed to assess long-term cytotoxicity and in vivo biodistribution and biodegradation.

\section{Acknowledgments}

The authors would like to thank the Nanotesla Institute Ljubljana for their technical support in the preparation of nanoparticles and Maruša Stražišar and Matej Skočaj for technical support. This work was supported by Slovenian Research Agency within project J2-2249 and Young Researchers Program.

\section{Disclosure}

The authors report no conflicts of interest in this work.

\section{References}

1. Stone V, Johnston H, Clift MJ. Air pollution, ultrafine and nanoparticle toxicology: cellular and molecular interactions. IEEE Trans Nanobioscience. 2007;6(4):331-340.

2. Oberdörster G, Oberdörster E, Oberdörster J. Nanotoxicology: An emerging discipline evolving from studies of ultrafine particles. Environ Health Perspect. 2005;113(7):823-839.

3. Salata O. Applications of nanoparticles in biology and medicine. J Nanobiotechnology. 2004;2(1):3.

4. Zhang L, Gu FX, Chan JM, Wang AZ, Langer RS, Farokhzad OC. Nanoparticles in medicine: therapeutic applications and developments. Clin Pharmacol Ther. 2008;83(5):761-769.

5. De M, Ghosh PS, Rotello VM. Applications of nanoparticles in biology. Adv Mater. 2008;20(22):4225-4241.

6. Pankhurst QA, Connolly J, Jones SK, Dobson J. Applications of magnetic nanoparticles in biomedicine. J Phys D Appl Phys. 2003;36(13): R167-R181.

7. Mahapatro A, Singh DK. Biodegradable nanoparticles are excellent vehicle for site directed in-vivo delivery of drugs and vaccines. J Nanobiotechnology. 2011;9(1):55.

8. Pankhurst QA, Thanh NKT, Jones SK, Dobson J. Progress in applications of magnetic nanoparticles in biomedicine. J Phys D Appl Phys. 2009;42(22):224001

9. Fröhlich E, Samberger C, Kueznik T, et al. Cytotoxicity of nanoparticles independent from oxidative stress. J Toxicol Sci. 2009;34(4): 363-375.

10. Lewinski N, Colvin V, Drezek R. Cytotoxicity of nanoparticles. Small. 2008;4(1):26-49.

11. Douglas KL, Piccirillo CA, Tabrizian M. Cell line-dependent internalization pathways and intracellular trafficking determine transfection efficiency of nanoparticle vectors. Eur J Pharm Biopharm. 2008;68(3): 676-687.

12. Harush-Frenkel O, Altschuler Y, Benita S. Nanoparticle-cell interactions: drug delivery implications. Crit Rev Ther Drug Carrier Syst. 2008;25(6):485-544.

13. Chavanpatil MD, Khdair A, Panyam J. Nanoparticles for cellular drug delivery: mechanisms and factors influencing delivery. $J$ Nanosci Nanotechnol. 2006;6(9-10):2651-2663.

14. Huang X, Teng X, Chen D, Tang F, He J. The effect of the shape of mesoporous silica nanoparticles on cellular uptake and cell function. Biomaterials. 2010;31(3):438-448.

15. Chithrani BD, Ghazani AA, Chan WC. Determining the size and shape dependence of gold nanoparticle uptake into mammalian cells. Nano Lett. 2006;6(4):662-668.

16. Wilhelm C, Gazeau F, Roger J, Pons JN, Bacri JC. Interaction of anionic superparamagnetic nanoparticles with cells: kinetic analyses of membrane adsorption and subsequent internalization. Langmuir. 2002;18(21):8148-8155.

17. Chithrani BD, Chan WC. Elucidating the mechanism of cellular uptake and removal of protein-coated gold nanoparticles of different sizes and shapes. Nano Lett. 2007;7(6):1542-1550.

18. Kim JS, Yoon TJ, Yu KN, et al. Cellular uptake of magnetic nanoparticle is mediated through energy-dependent endocytosis in A549 cells. $J$ Vet Sci. 2006;7(4):321-326.

19. Shukla R, Bansal V, Chaudhary M, Basu A, Bhonde RR, Sastry M. Biocompatibility of gold nanoparticles and their endocytotic fate inside the cellular compartment: a microscopic overview. Langmuir. 2005;21(23):10644-10654.

20. Suh H, Jeong B, Liu F, Kim SW. Cellular uptake study of biodegradable nanoparticles in vascular smooth muscle cells. Pharm Res. 1998;15(9):1495-1498.

21. Kumari S, Mg S, Mayor S. Endocytosis unplugged: multiple ways to enter the cell. Cell Res. 2010;20(3):256-275. 
22. Cartiera MS, Johnson KM, Rajendran V, Caplan MJ, Saltzman WM. The uptake and intracellular fate of PLGA nanoparticles in epithelial cells. Biomaterials. 2009;30(14):2790-2798.

23. Yen HJ, Hsu SH, Tsai CL. Cytotoxicity and immunological response of gold and silver nanoparticles of different sizes. Small. 2009;5(13): 1553-1561.

24. Soenen SJ, Nuytten N, De Meyer SF, De Smedt SC, De Cuyper M. High intracellular iron oxide nanoparticle concentrations affect cellular cytoskeleton and focal adhesion kinase-mediated signaling. Small. 2010;6(7):832-842.

25. AshaRani PV, Low Kah Mun G, Hande MP, Valiyaveettil S. Cytotoxicity and genotoxicity of silver nanoparticles in human cells. ACS Nano. 2009;3(2):279-290.

26. Stayton I, Winiarz J, Shannon K, Ma Y. Study of uptake and loss of silica nanoparticles in living human lung epithelial cells at single cell level. Anal Bioanal Chem. 2009;394(6):1595-1608.

27. Besic Gyenge E, Darphin X, Wirth A, et al. Uptake and fate of surface modified silica nanoparticles in head and neck squamous cell carcinoma. J Nanobiotechnology. 2011;9:32.

28. Moore A, Weissleder R, Bogdanov A Jr. Uptake of dextran-coated monocrystalline iron oxides in tumor cells and macrophages. J Magn Reson Imaging. 1997;7(6):1140-1145.

29. Busch W, Bastian S, Trahorsch U, et al. Internalisation of engineered nanoparticles into mammalian cells in vitro: influence of cell type and particle properties. J Nanopart Res. 2011;13(1):293-310.

30. Nam HY, Kwon SM, Chung H, et al. Cellular uptake mechanism and intracellular fate of hydrophobically modified glycol chitosan nanoparticles. J Control Release. 2009;135(3):259-267.

31. Yan Y, Johnston AP, Dodds SJ, et al. Uptake and intracellular fate of disulfide-bonded polymer hydrogel capsules for Doxorubicin delivery to colorectal cancer cells. ACS Nano. 2010;4(5):2928-2936.

32. Marmorato P, Ceccone G, Gianoncelli A, et al. Cellular distribution and degradation of cobalt ferrite nanoparticles in Balb/3T3 mouse fibroblasts. Toxicol Lett. 2011;207(2):128-136.

33. Xiao Y, Forry SP, Gao X, Holbrook RD, Telford WG, Tona A. Dynamics and mechanisms of quantum dot nanoparticle cellular uptake. J Nanobiotechnology. 2010;8:13.

34. Qi LF, Xu ZR, Li Y, Jiang X, Han XY. In vitro effects of chitosan nanoparticles on proliferation of human gastric carcinoma cell line MGC803 cells. World J Gastroenterol. 2005;11(33):5136-5141.

35. Berry CC, Wells S, Charles S, Aitchison G, Curtis AS. Cell response to dextran-derivatised iron oxide nanoparticles post internalisation. Biomaterials. 2004;25(23):5405-5413.

36. Asharani PV, Hande MP, Valiyaveettil S. Anti-proliferative activity of silver nanoparticles. BMC Cell Biol. 2009;10:65.

37. Campelj S, Makovec D, Drofenik M. Preparation and properties of water-based magnetic fluids. J Phys Condens Matter. 2008;20(20): 204101.

38. Tourinho FA, Franck R, Massart R. Aqueous ferrofluids based on manganese and cobalt ferrites. J Mater Sci. 1990;25(7):3249-3254.

39. Bregar VB, Pavlin M, Žnidaršič A. Magnetization state in magnetic nanoparticle agglomerates. Proceedings of the 8th International Conference on the Scientific and Clinical Applications of Magnetic Carriers; May 25-29, 2010; Rostock, Germany. Melville, NY: American Institute of Physics; 2010;1311(1):59-64.
40. Prijic S, Prosen L, Cemazar M, et al. Surface modified magnetic nanoparticles for immuno-gene therapy of murine mammary adenocarcinoma. Biomaterials. 2012;33(17): 4379-4391.

41. Pavlin M, Bregar VB. Stability of nanoparticle suspensions in different biologically relevant media. Dig J Nanomater Bios. 2012;7(4): 1389-1400.

42. Mercer J, Helenius A. Virus entry by macropinocytosis. Nat Cell Biol. 2009;11(5):510-520.

43. Kocbek P, Teskač K, Kreft ME, Kristl J. Toxicological aspects of longterm treatment of keratinocytes with $\mathrm{ZnO}$ and $\mathrm{TiO} 2$ nanoparticles. Small. 2010;6(17):1908-1917.

44. Roohi F, Lohrke J, Ide A, Schütz G, Dassler K. Studying the effect of particle size and coating type on the blood kinetics of superparamagnetic iron oxide nanoparticles. Int J Nanomedicine. 2012;7:4447-4458.

45. Ding D, Zhu Z, Liu Q, et al. Cisplatin-loaded gelatin-poly(acrylic acid) nanoparticles: synthesis, antitumor efficiency in vivo and penetration in tumors. Eur J Pharm Biopharm. 2011;79(1):142-149.

46. Hu Y, Ding Y, Ding D, et al. Hollow chitosan/poly(acrylic acid) nanospheres as drug carriers. Biomacromolecules. 2007;8(4):1069-1076.

47. Safi M, Sarrouj H, Sandre O, Mignet N, Berret J-F. Interactions between sub-10-nm iron and cerium oxide nanoparticles and 3T3 fibroblasts: the role of the coating and aggregation state. Nanotechnology. 2010;21(14): 145103 .

48. Quintart J, Leroy-Houyet MA, Trouet A, Baudhuin P. Endocytosis and chloroquine accumulation during the cell cycle of hepatoma cells in culture. J Cell Biol. 1979;82(3):644-653.

49. Fielding AB, Willox AK, Okeke E, Royle SJ. Clathrin-mediated endocytosis is inhibited during mitosis. Proc Natl Acad Sci U S A. 2012;109(17):6572-6577.

50. Swanson JA, Watts C. Macropinocytosis. Trends Cell Biol. 1995;5(11) 424- 428.

51. Verma A, Stellacci F. Effect of surface properties on nanoparticle-cell interactions. Small. 2010;6(1):12-21.

52. Di Virgilio AL, Reigosa M, Arnal PM, Fernández Lorenzo de Mele M. Comparative study of the cytotoxic and genotoxic effects of titanium oxide and aluminium oxide nanoparticles in Chinese hamster ovary (CHO-K1) cells. J Hazard Mater. 2010;177(1-3):711-718.

53. Dittrich C, Burckhardt CJ, Danuser G. Delivery of membrane impermeable cargo into $\mathrm{CHO}$ cells by peptide nanoparticles targeted by a protein corona. Biomaterials. 2012;33(9):2746-2753.

54. Vercauteren D, Vandenbroucke RE, Jones AT, et al. The use of inhibitors to study endocytic pathways of gene carriers: optimization and pitfalls. Mol Ther. 2010;18(3):561-569.

55. Ivanov AI. Pharmacological inhibition of endocytic pathways: is it specific enough to be useful? In: Ivanov AI, Walker JM, editors. Exocytosis and Endocytosis. Methods in Molecular Biology. Totowa: Humana Press; 2008;440:15-33.

56. Iversen TG, Skotland T, Sandvig K. Endocytosis and intracellular transport of nanoparticles: Present knowledge and need for future studies. Nano Today. 2011;6(2):176-185.

57. Luzio JP, Pryor PR, Bright NA. Lysosomes: fusion and function. Nat Rev Mol Cell Biol. 2007;8(8):622-632.

58. Panyam J, Labhasetwar V. Dynamics of endocytosis and exocytosis of poly(D,L-lactide-co-glycolide) nanoparticles in vascular smooth muscle cells. Pharm Res. 2003;20(2):212-220. 


\section{Supplementary material}
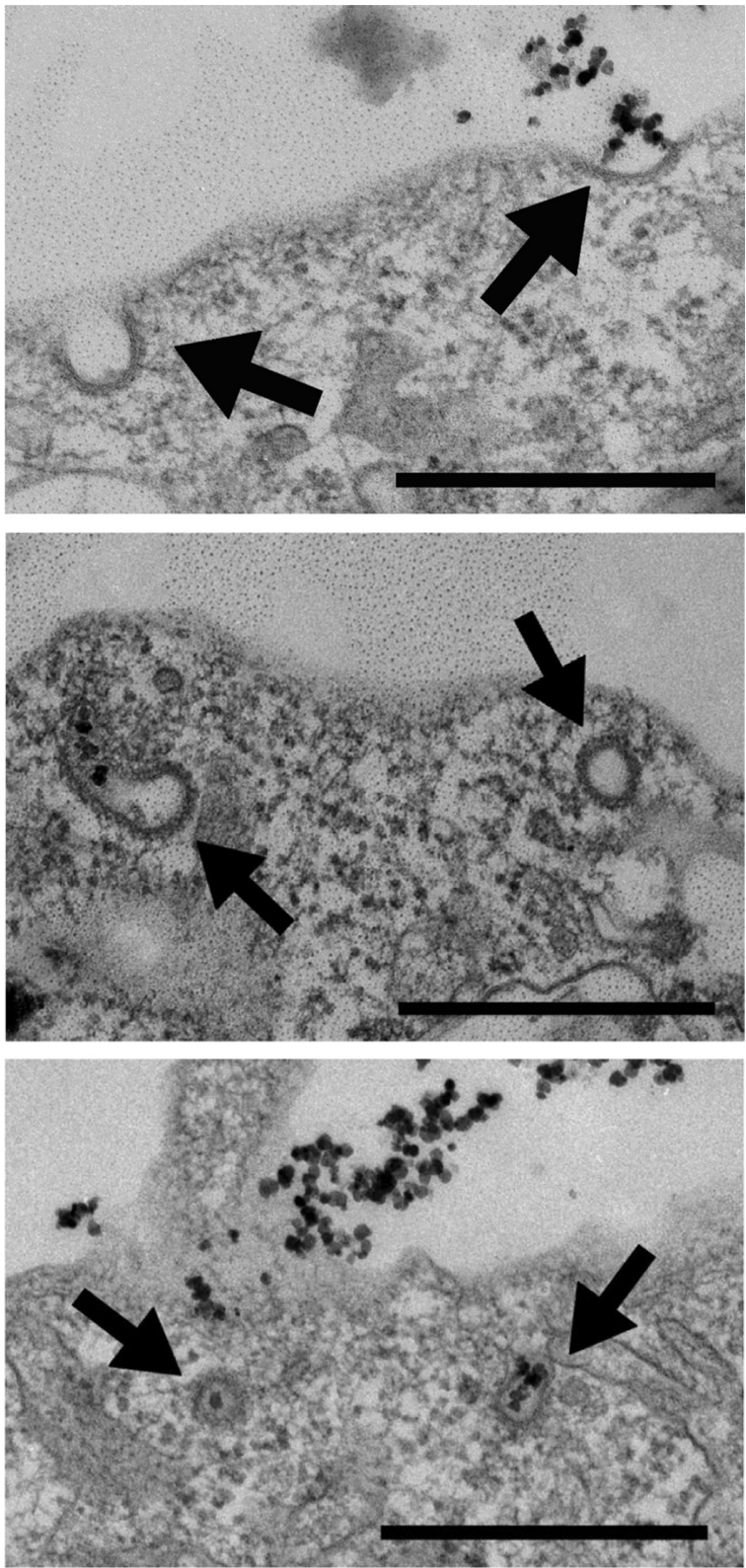

Figure SI Representative TEM micrographs of clathrin-coated pits and clathrin-coated vesicles observed in $\mathrm{CHO}$ cells incubated with $100 \mu \mathrm{g} / \mathrm{mL}$ for different incubation times, from 10 minutes to I hour.

Notes: Structures resembling clathrin-coated structures were observed during all observed time points and are denoted by arrows. The scale bars corespond to $0.5 \mu \mathrm{m}$

Abbreviations: TEM, transmission electron microscopy; $\mathrm{CHO}$, Chinese hamster ovary.

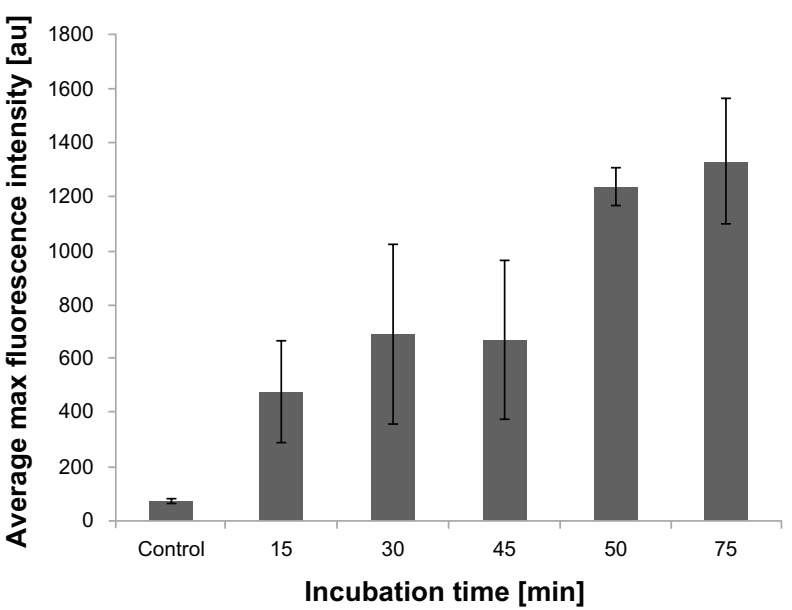

Figure S2 Average maximal fluorescence intensity increases with longer incubation times.

Notes: RITC was electrostatically bound to the PAA-functionalized NPs. Cells were exposed to NPs for increasing incubation times, and maximal fluorescence intensity was measured for separate cells. The control sample was incubated with the appropriate volume of the last dialysate water, containing the same amount of unbound RITC as the NP suspension after dialysis.

Abbreviations: RITC, rhodamine B isothiocyanate, PAA, polyacrylic acid; NP, nanoparticle. 


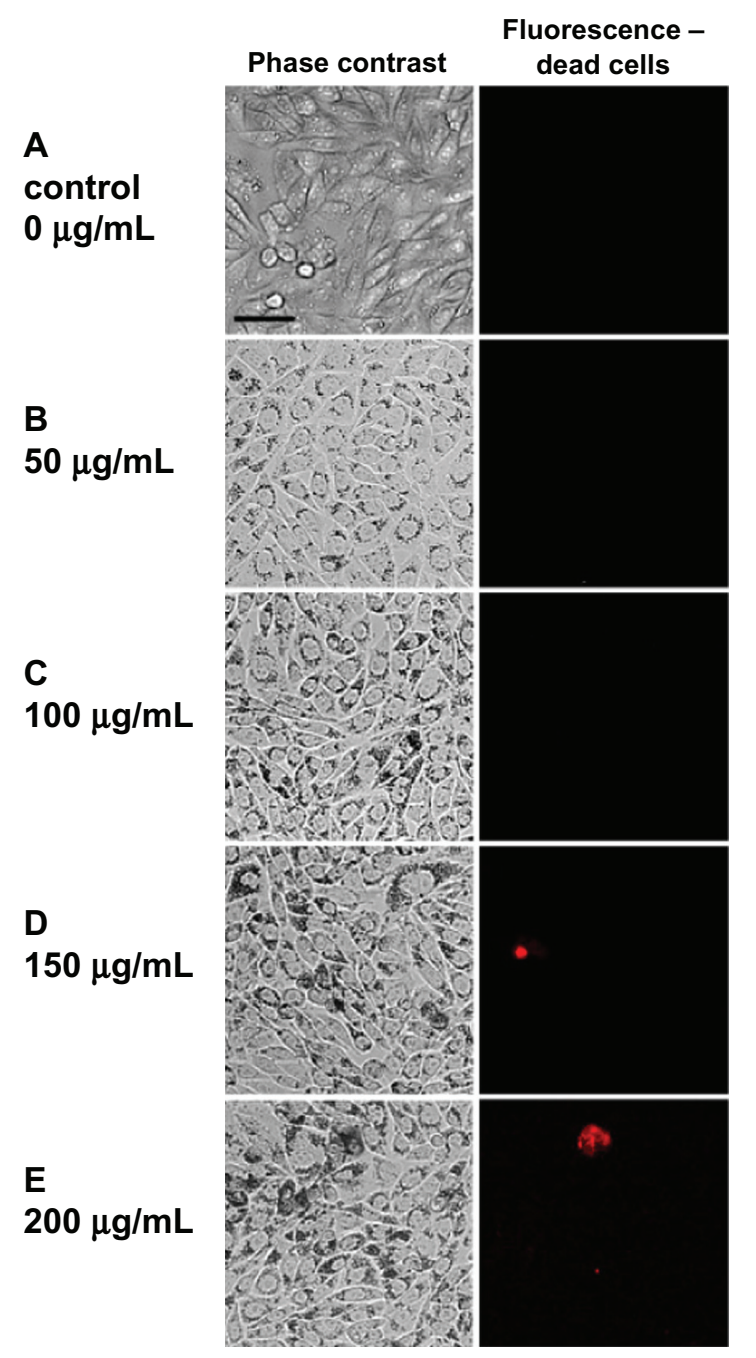

Figure S3 Observation of $\mathrm{CHO}$ cells after exposure to different NP concentrations $(0,50,100,150$, and $200 \mu \mathrm{g} / \mathrm{mL})$ for 24 hours.

Notes: For all samples, phase contrast was taken, and fluorescence images of the same cells stained with fluorescent dye propidium iodide, which only stains dead cells. In the phase-contrast images, large quantities of internalized nanoparticles can be observed arround the nucleus. No significant effect on viability and proliferation could be observed. Representative images are shown. The scale bar corresponds to $45 \mu \mathrm{m}$.

Abbreviations: $\mathrm{CHO}$, Chinese hamster ovary; NP, nanoparticle.

International Journal of Nanomedicine

\section{Publish your work in this journal}

The International Journal of Nanomedicine is an international, peerreviewed journal focusing on the application of nanotechnology in diagnostics, therapeutics, and drug delivery systems throughou the biomedical field. This journal is indexed on PubMed Central,

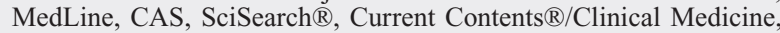

Journal Citation Reports/Science Edition, EMBase, Scopus and the Elsevier Bibliographic databases. The manuscript management system is completely online and includes a very quick and fair peer-review system, which is all easy to use. Visit http://www.dovepress.com/ testimonials.php to read real quotes from published authors.

\footnotetext{
Submit your manuscript here: http://www.dovepress.com/international-journal-of-nanomedicine-journal
} 